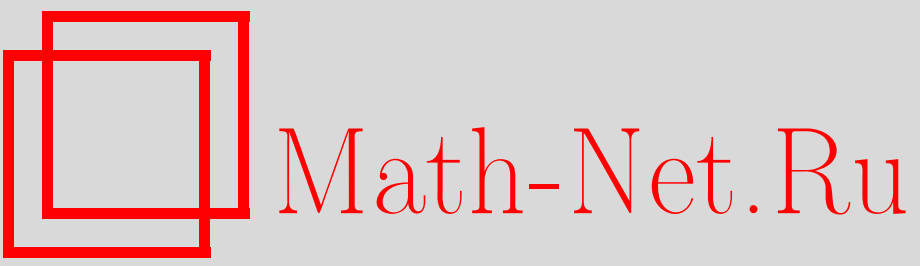

В. П. Радченко, В. А. Кирпичёв, В. В. Лунин, Влияние пневмодробеструйной обработки и термоэкспозиции на остаточные напряжения и предел выносливости образцов с надрезами из сплавов В95 и Д16Т, Вестн. Сам. гос. техн. ун-та. Сер. Физ.-мат. науки, 2011, выпуск 3(), 181-184

DOI: https://doi.org/10.14498/vsgtu994

Использование Общероссийского математического портала Math-Net.Ru подразумевает, что вы прочитали и согласны с пользовательским соглашением http: //www . mathnet.ru/rus/agreement

Параметры загрузки:

IP: 44.207 .124 .84

26 апреля 2023 г., $17: 06: 17$ 
УДК 621.787:539.319

\title{
ВЛИЯНИЕ ПНЕВМОДРОБЕСТРУЙНОЙ ОБРАБОТКИ И ТЕРМОЭКСПОЗИЦИИ НА ОСТАТОЧНЫЕ НАПРЯЖЕНИЯ И ПРЕДЕЛ ВЫНОСЛИВОСТИ ОБРАЗЦОВ С НАДРЕЗАМИ ИЗ СПЛАВОВ В95 И Д16Т
}

\author{
В.П. Радченко ${ }^{1}$ В.А. Кирпичёв ${ }^{2}$, В.В. Лунин ${ }^{1}$ \\ 1 Самарский государственный технический университет, \\ 443100, Самара, ул. Молодогвардейская, 244. \\ 2 Самарский государственный аэрокосмический университет им. ак. С. П. Королёва \\ (национальный исследовательский университет), \\ 443086, Самара, Московское ш., 34 . \\ E-mails: radch@samgtu.ru, sopromat@ssau.ru, luloon@mail.ru
}

\begin{abstract}
Экспериментально исследовано влияние пневмодробеструйной обработки и температурной выдержки без нагрузки (термоэкспозиции) на остаточные напряжения и предел выносливости гладких образцов и образцов с полукруглыми надрезами из алюминиевых сплавов В95 и Д16Т. Исследование выполнено для симметричного иикла нагружения в условиях изгиба и растяжения-сжатия и базы испытаний $-10^{7}$ ииклов. Выполнена проверка расчётного критерия среднеинтегралъных остаточных напряжений для оченки приращения предела выносливости упрочнёных деталей с концентраторами напряжений. Эксперименталъно и расчётным путём установлено увеличение приращения предела выносливости упрочнённых деталей с концентраторами напряжений по сравнению с неупрочнёнными образиами. Показано, что термоэкспозиция приводит к релаксации остаточных напряжений и снижению приращения предела выносливости. Наблюдается соответствие расчётных и экспериментальных данных.
\end{abstract}

Ключевые слова: поверхностное упрочнение, чилиндрический образеч, полукруглый надрез, остаточные напряжения, предел сопротивления усталости, температурная выдержка.

Для сокращения длительных и дорогостоящих испытаний на усталость при внедрении в производство новых упрочняющих технологий необходимо разрабатывать и апробировать научно обоснованные расчётные методы прогнозирования характеристик сопротивления усталости деталей. Известно, что основную роль в повышении сопротивления усталости поверхностно упрочнённых деталей в условиях концентрации играют сжимающие остаточные напряжения [1]. В работе [2] для оценки приращения предела выносливости упрочнённых деталей с концентраторами по остаточным напряжениям был предложен критерий среднеинтегральных остаточных напряжений $\bar{\sigma}_{\text {ост }}$ :

$$
\bar{\sigma}_{\mathrm{OCT}}=\frac{2}{\pi} \int_{0}^{1} \frac{\sigma_{z}(\xi)}{\sqrt{1-\xi^{2}}} d \xi,
$$

где $\sigma_{z}(\xi)$ - осевые остаточные напряжения в наименьшем сечении детали с концентратором; $t_{\text {кр }}$ - критическая глубина нераспространяющейся трещины усталости, возникающей при работе детали на пределе выносливости (феноменологический параметр); $\xi=a / t_{\text {кр }}$ - расстояние от дна концентратора до текущего слоя, выраженное в долях $t_{\text {кр }}$.

Приращение предела выносливости при изгибе и растяжении-сжатии в случае

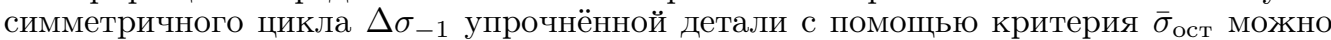

Владимир Павлович Радченко (д.ф.-м.н., проф.), зав. кафедрой, каф. прикладной математики и информатики. Виктор Алексеевич Кирпичёв (д.т.н., доц.), декан, факультет летательных аппаратов. Валентин Валериевич Лунин, аспирант, каф. прикладной математики и информатики. 
определить по формуле

$$
\Delta \sigma_{-1}=\bar{\psi}_{\sigma}\left|\bar{\sigma}_{\text {ост }}\right|
$$

где $\bar{\psi}_{\sigma}$ - феноменологический коэффициент влияния остаточных напряжений на предел выносливости.

Для проверки возможности применения критерия $\bar{\sigma}_{\text {ост }}$ в случае поверхностного упрочнения деталей из алюминиевых сплавов были проведены эксперименты на образцах с надрезами из материалов В95 и Д16Т.

Гладкие образцы диаметром 15 мм с отверстием 5 мм подвергались пневмодробеструйной обработке (ПДО) дробью диаметром 1,5-2 мм при давлении воздуха 0,25 МПа в течение 10 минут. Затем на половину упрочнённых и неупрочнённых гладких образов наносились круговые надрезы полукруглого профиля двух радиусов: $R=0,3$ мм и $R=0,5$ мм. Остаточные напряжения в гладких образцах определялись экспериментально методом колец и полосок [3], а в образцах с надрезом - суммированием дополнительных напряжений, возникающих при нанесении надрезов на упрочнённую поверхность, с остаточными напряжениями гладких образцов [4]. Распределение осевых остаточных напряжений $\sigma_{z}$ по толщине поверхностного слоя $a$ в гладких и надрезанных образцах представлено на рис. 1.

Испытания образцов с надрезом на усталость при чистом изгибе в случае симметричного цикла проводились на машинах МУИ-6000, база испытаний - $10^{7}$ циклов нагружения. Результаты определения предела выносливости $\sigma_{-1}$, измерения $t_{\text {кр }}$, расчёта $\bar{\sigma}_{\text {ост }}$ и коэффициента $\bar{\psi}_{\sigma}$ представлены в табл. 1.

Из представленных в табл. 1 данных видно, что для упрочнённых образцов с надрезом из алюминиевых сплавов В95 и Д16Т значение коэффициента $\vec{\psi}_{\sigma}$ при изгибе в случае симметричного цикла составляет в среднем 0,373 , что практически совпадает со значением $\bar{\psi}_{\sigma}$ для образцов и деталей из сталей и сплавов на основе никеля с такой же концентрацией напряжений [5].

Половина упрочнённых и неупрочнённых образцов подвергалась термоэкспозиции при температуре $T=125^{\circ} \mathrm{C}$ в течение 100 часов. Далее на гладкие образцы
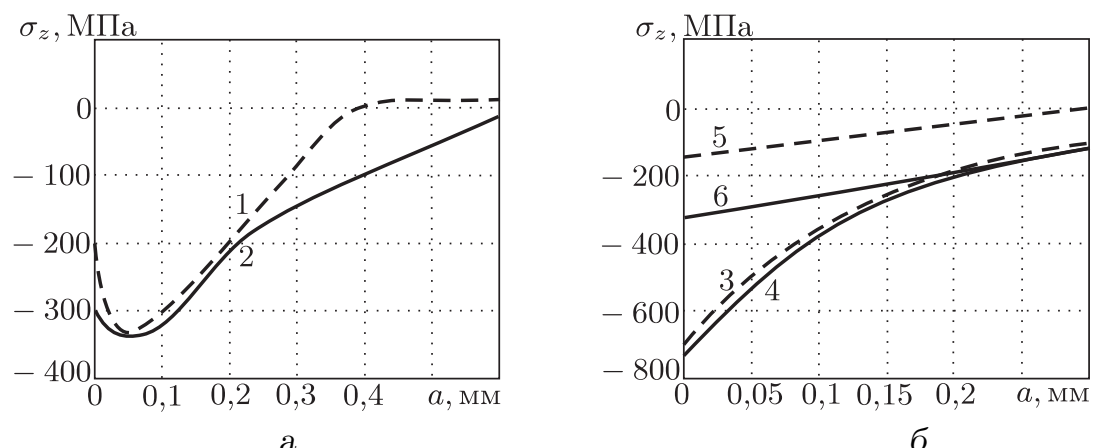

Рис. 1. Осевые остаточные напряжения $\sigma_{z}$ в упрочнённых ПДО гладких образцах (а) и в образцах с надрезом (б) из сплавов $\mathrm{B} 95(1,3,5)$ и Д16Т $(2,4,6): 3,4-R=0,3$ мм; $5,6-$ $R=0,5 \mathrm{Mм}$

Таблица 1

Результаты испытаний образцов после ПДО

\begin{tabular}{c|c|c|c|c|c|c}
\hline \multirow{2}{*}{ Материал } & Радиус & Надреза & \multirow{2}{*}{$\begin{array}{c}\text { обпрочнёцы } \\
\text { обрые }\end{array}$} & \multicolumn{4}{|c}{ Упрочнённые образцы } \\
\cline { 4 - 7 } & $R$, мм & $\sigma_{-1}$, МПа & $\begin{array}{c}\sigma_{-1}, \\
\text { МПа }\end{array}$ & $\begin{array}{c}t_{\text {кр }}, \\
\text { мм }\end{array}$ & $\begin{array}{c}\bar{\sigma}_{\text {ост, }}, \\
\text { МПа }\end{array}$ & $\bar{\psi}_{\sigma}$ \\
\hline \multirow{2}{*}{ В95 } & 0,3 & 105 & 200 & 0,310 & -249 & 0,382 \\
& 0,5 & 107,5 & 132,5 & 0,320 & $-72,2$ & 0,347 \\
\hline \multirow{2}{*}{ Д16Т } & 0,3 & 82,5 & 182,5 & 0,300 & -261 & 0,383 \\
& 0,5 & 82,5 & 157,5 & 0,320 & -197 & 0,381 \\
\hline
\end{tabular}


наносились надрезы полукруглого профиля двух радиусов: $R=0,3$ мм и $R=0,5$ мм.

Остаточные напряжения в образцах определялись так же, как и в образцах до термоэкспозиции. Эпюры осевых остаточных напряжений в стандартной цилиндрической системе координат по толщине поверхностного слоя $a$ в гладких и надрезанных образцах после термоэкспозиции представлены на рис. 2. Из приведённого на рис. 2 распределения остаточных напряжений видно, что после термоэкспозиции в образцах из обоих сплавов происходит релаксация остаточных напряжений, при этом в образцах из сплава В95 сжимающие остаточные напряжения значительно меньше, чем в образцах из сплава Д16Т.

Испытания на усталость образцов с надрезом при чистом изгибе в случае симметричного цикла проводились при $T=20^{\circ} \mathrm{C}$ также на машинах МУИ-6000, база испытаний $-10^{7}$ циклов нагружения. Результаты испытаний на усталость и определения остаточных напряжений представлены в табл. 2.

Из приведённых в таблл. 2 данных можно видеть, что коэффициент влияния остаточных напряжений $\bar{\psi}_{\sigma}$ на предел выносливости по критерию $\bar{\sigma}_{\text {ост }}$ составляет в среднем 0,368 и близок к значению $\bar{\psi}_{\sigma}=0,36$, установленному в [5] для упрочнённых образцов с такой же степенью концентрации напряжений.

Таким образом, проведённое исследование показывает, что критерий среднеин-

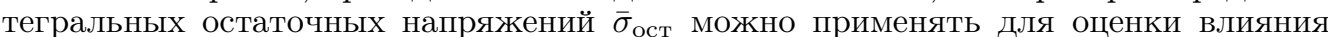
поверхностного упрочнения и последующего температурного воздействия на предел выносливости образцов с концентраторами, изготовленными из алюминиевых сплавов В95 и Д16Т.
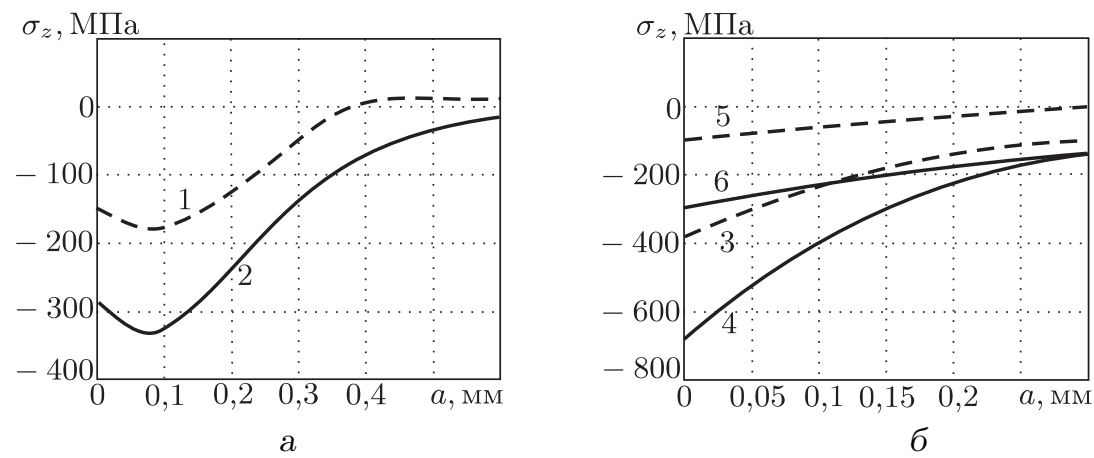

Рис. 2. Осевые остаточные напряжения $\sigma_{z}$ после ПДО и термоэкспозиции в гладких образцах (а) и в образцах с надрезом (б) из сплавов В95 $(1,3,5)$ и Д16Т $(2,4,6): 3,4-$ $R=0,3$ мм; $5,6-R=0,5$ мм

Таблица 2

Результаты испытаний образцов после ПДО и термоэкспозиции

\begin{tabular}{|c|c|c|c|c|c|c|}
\hline \multirow[b]{2}{*}{ Материал } & \multirow{2}{*}{$\begin{array}{c}\text { Радиус } \\
\text { надреза } \\
R, \text { мм }\end{array}$} & \multirow{2}{*}{$\begin{array}{c}\text { Неупрочнённые } \\
\text { образцы } \\
\sigma_{-1}, \text { МПа } \\
\end{array}$} & \multicolumn{4}{|c|}{ Упрочнённые образцы } \\
\hline & & & $\begin{array}{l}\sigma_{-1}, \\
\text { МПа }\end{array}$ & $\begin{array}{c}t_{\mathrm{Kp}}, \\
\mathrm{MM}\end{array}$ & $\begin{array}{l}\bar{\sigma}_{\text {ост }} \\
\text { МПа }\end{array}$ & $\psi_{\sigma}$ \\
\hline \multirow{2}{*}{ B95 } & 0,3 & 105 & 155 & 0,310 & -141 & 0,355 \\
\hline & 0,5 & 107,5 & 115 & 0,320 & $-21,3$ & 0,352 \\
\hline \multirow{2}{*}{ Д16Т } & 0,3 & 82,5 & 175 & 0,320 & -250 & 0,370 \\
\hline & 0,5 & 82,5 & 150 & 0,310 & -171 & 0,395 \\
\hline
\end{tabular}

Работа выполнена при поддержке Министерства образования и науки РФ в рамках АВЦП «Развитие научного потенциала высшей школы» (проект 2.1.1/13944).

БИБЛИОГРАФИЧЕСКИЙ СПИСОК

1. Иванов С. И., Павлов В.Ф. Влияние остаточных напряжений и наклёпа на усталостную прочность // Пробл. прочности, 1976. №5. С. 25-27; англ. пер.: Ivanov S. I. Pavlov V. F. 
The effect of residual stresses and cold working on fatigue strength // Strength of Materials. Vol. 8, no. 5. Pp. 529-531.

2. Павлов В.Ф. О связи остаточных напряжений и предела выносливости при изгибе в условиях концентрации напряжений // Изв. вузов. Машиностроение, 1986. № 8. С. 29 32. [Pavlov V.F. The relation between residual stress and fatigue limit in case of bending in conditions of stress concentration // Izv. vuzov. Mashinostroenie, 1986. no. 8. Pp. 29-32].

3. Иванов С.И. К определению остаточных напряжений в цилиндре методом колец и полосок / В сб.: Остаточные напряжения. Вып. 53. Куйбышев: КуАИ, 1971. С. 32-42. [Ivanov S.I. Determination of residual stresses in a cylinder by the ring and band method / In: Residual Stresses. Issue 53. Kuibyshev: KuAI, 1971. Pp. 32-42].

4. Иванов С.И., Шатунов М. П., Павлов В.Ф. Влияние остаточных напряжений на выносливость образцов с надрезом / В сб.: Вопросы прочности элементов авиачионных конструкиий. Вып. 1. Куйбышев: КуАИ, 1974. С. 88-95. [Ivanov S. I., Shatunov M. P., Pavlov V.F. Effect of residual stresses on the fatigue of noth specimens / In: Problems of aircraft structure element strength. Issue 1. Kuibyshev: KuAI, 1974. Pp. 88-95].

5. Павлов В.Ф., Кирпичёв В.А., Иванов В.Б. Остаточные напряжения и сопротивление усталости упрочнённых деталей с концентраторами напряжений. Самара: СНЦ РАН, 2008. 64 c. [Pavlov V.F., Kirpichyov V.A., Ivanov V.B. Residual stresses and fatigue resistance of strengthened details with stress concentrators. Samara: SNC RAN, 2008. 64 pp.]

Поступила в редакцию 18/VII/2011;

в окончательном варианте - 04/IX/2011.

MSC: 74R10; 74G70, 74-05

\title{
INFLUENCE OF AIR SHOT BLASTING AND TEMPERATURE EXPOSURE ON RESIDUAL STRESSES AND FATIGUE STRENGTH OF NOTCHED SPECIMENS FROM ALLOYS V95 AND D16T
}

\author{
V.P. Radchenko ${ }^{1}$, V.A. Kirpichyov ${ }^{2}$, V. V. Lunin ${ }^{1}$ \\ 1 Samara State Technical University, \\ 244, Molodogvardeyskaya st., Samara, 443100, Russia. \\ 2 S. P. Korolyov Samara State Aerospace University \\ (National Research University), \\ 34, Moskovskoe sh., Samara, 443086, Russia. \\ E-mails: radch@samgtu.ru, sopromat@ssau.ru, luloon@mail.ru
}

The effect of air shot blasting and temperature exposure without load on residual stresses and limit endurance of smooth specimens and specimens with semicircular notches of aluminum D16T and V95 alloys was investigated experimentally. This research was done for the symmetric loading cycle in terms of bending and tensioncompression tests and the base of $10^{7}$ cycles. The check of the calculated mean integral residual criterion stress to estimate the increment of the endurance limit of hardened parts with stress concentrators was completed. It was established experimentally and theoretically, that there is increment of increase in endurance limit hardened parts with stress concentrators in comparison with non-reinforced specimens, and temperature exposure leads to residual stresses relaxation and reduce the increment of the endurance limit.

Key words: surface hardening, cylindrical sample, semicircular cut, residual stresses, limit of resistance fatigue, temperature exposure.

Original article submitted 18/VII/2011; revision submitted 04/IX/2011.

Vladimir P. Radchenko (Dr. Sc. (Phys. \& Math.)), Head of Dept., Dept. of Applied Mathematics \& Computer Science. Victor A. Kirpichyov (Dr. Sc. (Techn.)), Dean, Faculty of Aircraft Construction. Valentin V. Lunin, Postgraduate Student, Dept. of Applied Mathematics \& Computer Science. 